\title{
Correction to: Implementation of a Respiratory Disease Forecasting Model Using LSTM for Central Taiwan
}

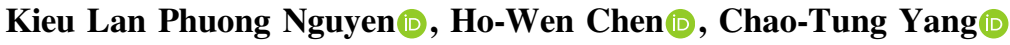
and Endah Kristiani $(\mathbb{D}$

\section{Correction to:}

Chapter "Implementation of a Respiratory Disease Forecasting Model Using LSTM for Central Taiwan" in: K. J. Kim and H.-Y. Kim (eds.), Information Science and Applications, Lecture Notes in Electrical Engineering 621, https://doi.org/10.1007/978-981-15-1465-4_44

In the original version of the book, the chapter "Implementation of a Respiratory Disease Forecasting Model Using LSTM for Central Taiwan" was published with the wrong affiliation for the author "Ho-Wen Chen". The chapter and book have been updated with the changes. 